Communications in Physics, Vol.21, No. 1 (2011), pp. 77-82

\title{
STUDY OF MELTING TEMPERATURE OF METALS: PRESSURE DEPENDENCE
}

\author{
VU VAN HUNG AND HOANG VAN TICH \\ Hanoi National University of Education \\ DANG THANH HAI \\ Vietnam Education Publishing House
}

\begin{abstract}
The melting temperature of metals are studied using the statistical moment method, going the quasi-harmonic appromations. The melting temperature of metals are calculated as a function of the pressure. We discuss the pressure dependence of melting temperatures of Ce, $\mathrm{Fe}$ metals and compare the present results with those of the experimental results.
\end{abstract}

\section{INTRODUCTION}

High-pressure behaviour of materials is of great interest from the viewpoints of geophysics [1] as well as of solid states sciences and technologies. The study of the phase stabilities and phase diagrams under extreme (high-pressure) conditions has also become quite interesting in recent years since the many geophysical and technological applications. It is the purpose of the present paper to study the pressure dependence of the melting temperatures of metals by the basis on the statistical moment method (SMM) [2-6].

In order to determine theoretically the melting temperatures of metals we will use the equilibrium condition of the solid phases. In particular, we will use the limiting condition for the absolute crystalline stability in order to find the melting temperatures $T_{m}$ under the hydrostatic pressure. Rigorously speaking, melting temperatures of the solid phases are defined as the temperature points at which the solid and the liquid states coexist in the thermally equilibrium condition [7]. Since the treatments of liquid phases are rather complicated [1-8], the most of the previous studies have been performed on the basis of the properties of the solid phases (starting with the Lindermann's formula) theorized in terms of the lattice instability $[10,11]$, free energy of dislocation motions, or a simple order-disorder transition [7].

In the present study, we use the SMM to investigate the equation of state and melting temperatures of metals. We will calculate the pressure dependence of the nearest neighbor distance and the melting temperature of metals. 


\section{EQUATION OF STATES AND MELTING TEMPERATURES OF METALS BY STATISTICAL MOMENT METHOD}

\section{II.1. Pressure versus volume relation}

The pressure versus volume relation of the lattice is $[2,3]$

$$
P v=-a\left[\frac{1}{6} \frac{\partial U_{0}}{\partial a}+\theta x \operatorname{coth} x \frac{1}{2 k} \frac{\partial k}{\partial a}\right]
$$

where $x=\frac{\hbar \omega}{2 \theta}, \theta=k_{B} T, \omega$ is the vibrational frequencies of atoms, $P$ denotes the hydrostatic pressure and $v$ is the atomic volume $v=\frac{V}{N}$ of the crystal, being $v=\frac{4}{3 \sqrt{3}} a^{3}$ for the bcc lattice. Using Eq.(1), one can find the nearest neighbor distance $a$ at pressure $P$ and temperature $T$. However, for numerical calculations, it is convenient to determine firstly the nearest neighbor distance $a(P, 0)$ at pressure $P$ and at absolute zero temperature $T=0$. For $T=0$ temperature, Eq.(1) is reduced to

$$
P v=-a\left[\frac{1}{6} \frac{\partial U_{0}}{\partial a}+\frac{\hbar \omega_{0}}{4 k} \frac{\partial k}{\partial a}\right]
$$

For simplicity, we take the effective pair interaction energy in metal systems as the power law, similar to the Lennard - Jones

$$
\varphi(r)=\frac{D}{(n-m)}\left[m\left(\frac{r_{0}}{r}\right)^{n}-n\left(\frac{r_{0}}{r}\right)^{m}\right],
$$

where $D, r_{0}$ are determined to fit to the experimental data (e.g., cohesive energy and elastic modulus). For bcc metals we take into account the first nearest, second, third, fourth and fifth nearest neighbor interactions. Using the effective pair potentials of Eq.(3), it is straightforward to get the interaction energy $U_{0}$ and the parameter $k$ in the crystal as

$$
\begin{aligned}
U_{0} & =\frac{D}{(n-m)}\left[m A_{n}\left(\frac{r_{0}}{a}\right)^{n}-n A_{m}\left(\frac{r_{0}}{a}\right)^{m}\right] \\
k & =\frac{1}{2} \sum_{i}\left(\frac{\partial^{2} \varphi_{i 0}}{\partial u_{i \beta}^{2}}\right)_{e q} \\
& =\frac{D n m}{2 a^{2}(n-m)}\left\{\left[(n+2) A_{n+4}^{a_{i x}^{2}}-A_{n+2}\right]\left(\frac{r_{0}}{a}\right)^{n}-\left[(m+2) A_{m+4}^{a_{i x}^{2}}\right]\left(\frac{r_{0}}{a}\right)^{m}\right\} \\
& =m_{0} \omega_{0}^{2},
\end{aligned}
$$

where $m_{0}$ is the mass of particle, $\omega_{0}$ is the frequency of lattice vibration, and Eq.(2) can be transformed to the form for bcc and fcc metals:

$$
\begin{aligned}
P \frac{4}{3 \sqrt{3}} r_{0}^{3} & =c_{1} y^{n+3}-c_{2} y^{m+3}+\frac{c_{3} y^{n+4}-c_{4} y^{m+4}}{\sqrt{c_{5} y^{n}-c_{6} y^{m}}}, \\
P \frac{r_{0}^{3}}{\sqrt{2}} & =c_{1} y^{n+3}-c_{2} y^{m+3}+\frac{c_{3} y^{n+4}-c_{4} y^{m+4}}{\sqrt{c_{5} y^{n}-c_{6} y^{m}}},
\end{aligned}
$$


where $y=\frac{r_{0}}{a}$,

$$
\begin{aligned}
c_{1} & =A_{n} \frac{D n m}{6(n-m)} \\
c_{2} & =A_{m} \frac{D n m}{6(n-m)} \\
c_{3} & =\frac{\hbar}{4 \sqrt{m_{0}}} \sqrt{\frac{D n m}{2(n-m)}}(n+2)\left[(n+2) A_{n+4}^{a_{i x}^{2}}-A_{n+2}\right] \frac{1}{r_{0}} \\
c_{4} & =\frac{\hbar}{4 \sqrt{m_{0}}} \sqrt{\frac{D n m}{2(n-m)}}(m+2)\left[(m+2) A_{m+4}^{a_{i x}^{2}}-A_{m+2}\right] \frac{1}{r_{0}} \\
c_{5} & =(n+2) A_{n+4}^{a_{i x}^{2}}-A_{n+2} \\
c_{6} & =(m+2) A_{m+4}^{a_{i x}^{2}}-A_{m+2}
\end{aligned}
$$

where $A_{n}, A_{m} \ldots$ are the structural sums for the given crystal and defined by

$$
A_{n}=\sum_{i} \frac{Z_{i}}{\nu_{i}^{n}} ; \quad A_{m}=\sum_{i} \frac{Z_{i}}{\nu_{i}^{m}} ; \quad A_{n}^{a_{i x}^{2}}=\frac{1}{a^{2}} \sum_{i} \frac{Z_{i x} a_{i x}^{2}}{\nu_{i}^{n}}
$$

here $Z_{i}$ is the coordination number of $i^{t h}$ nearest neighbor atoms with radius $r_{i}$ (for bcc lattice $r_{k}=\nu_{k} a_{0} ; \nu_{1}=1, Z_{1}=8 ; \nu_{2}=\sqrt{\frac{4}{3}}, Z_{2}=6 ; \nu_{3}=\sqrt{\frac{8}{3}}, Z_{3}=12 ; \nu_{4}=\sqrt{\frac{11}{3}}, Z_{4}=24$; ...).

In principle Eqs. $(6,7)$ permits to find the nearest neighbor distance $a(P, 0)$ at zero temperature and pressure $P$. Using the Maple program and the values of parameters $D$ and $r_{0}$ determined by the experimental data [12]. Eqs. $(6,7)$ can be solved, we find the values of the nearest neighbor distance $a(P, 0)$ at temperature $T=0$ and pressure $P$. Calculated results for the nearest neighbor distance $a(P, 0)$ of Ce, Dy and Fe metals at zero temperature and pressure $P$ are presented in the Table 1 .

\section{II.2. Pressure versus volume relation}

In this subsection, we discuss the pressure dependence of melting temperatures of metals by the basis on the statistical moment method. The expression of the pressure $P$ (1) can be write in the form

$$
P=-\frac{a}{6 v} \frac{\partial U_{0}}{\partial a}+\frac{3 \gamma_{G} \theta}{v},
$$

where the Grüneisen constant $\gamma_{G}$ is given by

$$
\gamma_{G}=-\frac{a}{6 k} \frac{\partial k}{\partial a} x \operatorname{coth} x
$$


From the limiting condition of the absolute stability for the crystalline phase

$$
\left(\frac{\partial P}{\partial V}\right)_{T}=0, \quad i e .,\left(\frac{\partial P}{\partial a}\right)_{T}=0 .
$$

One can find the limiting temperature $T_{S}$ for the crystalline stability. This limiting temperature $T_{S}$ can be give in the third order approximation as

$$
T_{S} \approx T_{S}(0)+\left(\frac{\partial T}{\partial P}\right)_{a} P+\frac{1}{2}\left(\frac{\partial^{2} T}{\partial P^{2}}\right)_{a} P^{2}+\frac{1}{6}\left(\frac{\partial^{3} T}{\partial P^{3}}\right)_{a} P^{3}+\ldots
$$

where

$$
T_{S}(0)=\frac{a}{18 k_{B} \gamma_{G}} \frac{\partial U_{0}}{\partial a}
$$

In deriving Eq.(12), the thermodynamic relation $\left(\frac{\partial P}{\partial T}\right)_{a}\left(\frac{\partial T}{\partial V}\right)_{P}\left(\frac{\partial V}{\partial P}\right)_{T}=-1$ is used. From the equation of states (9), it is easy to find the following expressions

$$
\begin{aligned}
\left(\frac{\partial T}{\partial P}\right)_{a} & =\frac{v P}{3 k_{B} \gamma_{G}}\left[1-\frac{T}{\gamma_{G}}\left(\frac{\partial \gamma_{G}}{\partial T}\right)_{a}\right] \\
\left(\frac{\partial^{2} T}{\partial P^{2}}\right)_{a} & \approx-\frac{v^{2}}{\left(3 k_{B} \gamma_{G}\right)^{2}} \frac{1}{T}+\frac{v}{3 k_{B} \gamma_{G}} \frac{1}{T}\left(\frac{\partial T}{\partial P}\right)_{a} \\
\left(\frac{\partial^{3} T}{\partial P^{3}}\right)_{a} & \approx\left(\frac{v}{3 \theta \gamma_{G}}\right)^{2}\left(\frac{\partial T}{\partial P}\right)_{a}+\frac{v}{3 \theta \gamma T}\left[T\left(\frac{\partial^{2} T}{\partial P^{2}}\right)_{a}-\left(\frac{\partial T}{\partial P}\right)_{a}^{2}\right]
\end{aligned}
$$

From the Eqs.(12), (14), (15) and (16), we find the expression of the limiting temperature $T_{S}$ as

$$
\begin{gathered}
T_{S}(P) \approx T_{S}(0)+\frac{P v}{3 k_{B} \gamma_{G}}\left\{1-\frac{T_{S}(0)}{\gamma_{G}}\left(\frac{\gamma_{G}}{\partial T}\right)_{a}\left[1+\frac{P v}{6 k_{B} T_{S}(0) \gamma_{G}}\right]\right\}+ \\
+\left(\frac{v}{3 k_{B} \gamma_{G}}\right)^{2} \frac{P^{3}}{6 T_{S}^{2}(0)}\left(\frac{\partial T}{\partial P}\right)_{a}-\frac{P^{3} v}{18 k_{B} \gamma_{G} T_{S}^{2}(0)}\left[\left(\frac{v}{3 k_{B} \gamma_{G}}\right)^{2}+\left(\frac{\partial T}{\partial a}\right)_{a}^{2}\right] .
\end{gathered}
$$

After the nearest neighbor distance $a$ is known [2-4], we can find both harmonic and inharmonic vibration parameters $k, \omega, \frac{\partial k}{\partial a}, \frac{\partial U_{0}}{\partial a}, \ldots$ Therefore, Eq.(13) permits us to determine the limiting temperature of absolute stability $T_{S}(0)$ at pressure $P=0$.

The above Eq.(17) can be used to find the limiting temperature $T_{S}(P)$ at pressure $P$ in an interactive manner: Firstly, we calculate the limiting temperature at zero pressure $T_{S}(0)$. Then, $T_{S}(0)$ value is used to calculate $\gamma_{G}\left(T_{S}(0), P\right)$ and at pressure $P$ and limiting temperature $T_{S}(0)$ and the limiting temperature $T_{S}(P)$ is obtained by using the right hand side of Eq.(17). 


\section{RESULTS AND DISCUSSIONS}

In order to check the validity of the present SMM for the study of the melting temperature of the metallic systems described herein, we performed calculations for pure rare earth metals Ce and Fe. When the SMM is used to evaluate the limiting temperature of crystalline materials, it is very important to satisfy the self consistency conditions between the equilibrium lattice spacing, the related thermodynamic quantities $\gamma_{G}$ and $U_{0}$. In such a calculation, the melting temperature $T_{m}$ is approximated quite well by the limiting temperature $T_{S}$

$$
T_{m} \approx T_{S}
$$

Table 1: Calculated results for the nearest neighbor distance a $(P, 0)$ at zero temperature and pressure $P$

\begin{tabular}{cccccc}
\hline$P(G P a)$ & 0 & 5 & 10 & 20 & 30 \\
\hline$C e$ & 3.0445 & 2.9619 & 2.8909 & 2.7946 & 2.7279 \\
\hline$D y$ & 3.1571 & 3.0861 & 3.0353 & 2.9630 & 2.9108 \\
\hline$F e$ & 2.4318 & 2.4227 & 2.4144 & 2.3996 & 2.3753 \\
\hline
\end{tabular}

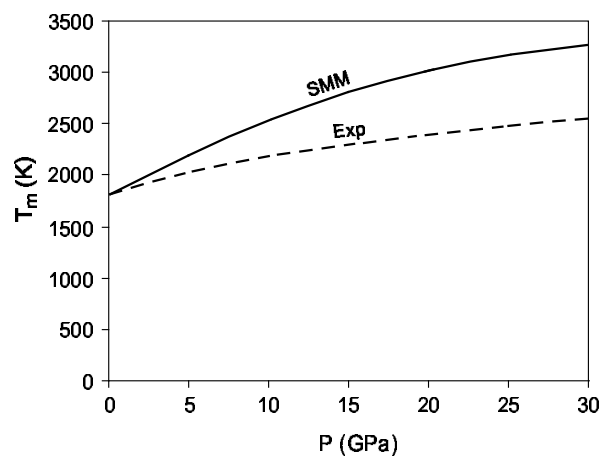

Fig.1. Melting temperatures of Fe

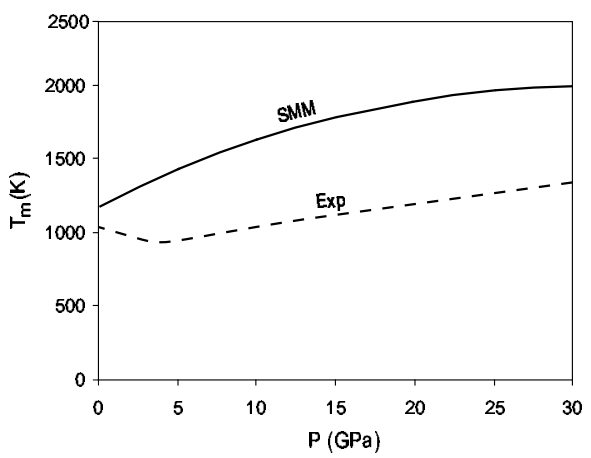

Fig.2. Melting temperatures of Ce

In Fig. 1 we present the pressure dependence of the melting (limiting) temperatures of Fe and in Fig.2 present the pressure dependence of the melting (limiting) temperatures of Ce, by solid lines, in comparison with the corresponding experimental results, by dashed lines. One can see in Fig.1, that the melting temperatures of Fe increase with the hydrostatic pressure, in agreement with the experimental results [13].

Table 2: Calculated pressure dependence of nearest neighbor distance $a\left(P, T_{S}\right)(\AA)$ and melting temperature $T_{m}(K)$ for Fe metal.

\begin{tabular}{cccccccc}
\hline$P(G P a)$ & 0 & 1 & 2 & 3 & 4 & 5 & 6 \\
\hline$a(P, 0)$ & 2.4318 & 2.4299 & 2.4281 & 2.4263 & 2.4245 & 2.4227 & 2.4210 \\
\hline$a\left(P, T_{S}\right)$ & 2.4772 & 2.4745 & 2.4720 & 2.4695 & 2.4670 & 2.4645 & 2.4622 \\
\hline$T_{S}(P)$ & 1810.0 & 1888.2 & 1965.9 & 2042.9 & 2118.9 & 2194.0 & 2267.8 \\
\hline $\operatorname{Exp}[13]$ & 1812.0 & 1873.0 & 1908.0 & 1943.0 & 1978.0 & 2013.0 & 2033.0 \\
\hline
\end{tabular}


One can see in Table 2 that the calculated pressure dependence, increasing rates, of the melting temperatures for Fe metal are very sensitive to the material, in good agreement with the corresponding experimental results [14].

\section{CONCLUSION}

The SMM calculations are perform by using the effective pair potential for the Fe, Ce and Dy metals. The use of the simple potentials is due to the fact that the purpose of the present study is to gain a general understanding of the effects of the inharmonic of the lattice vibration and pressure on the melting temperature for the bcc and fcc metals. The melting temperatures of Ce, Dy bcc metals and Fe fcc metal at a wide range of pressure calculated by using the SMM are generally in good agreement with the experimental results.

\section{REFERENCES}

[1] Luedemann H.D. and Kenedy G.C., J. Geophys. Res., 73 (1968) 2795

[2] Tang N. and Hung Vu Van, Phys. Stat. Sol.(b) 149 (1988) 511; 161 (1990) 165.

[3] Tang N. and Hung Vu Van, Phys. Stat. Sol.(b) 162 (1988) 379.

[4] Hung Vu Van and Masuda-Jindo K.,J. Phys. Soc. Jap. 69 (7) (2000) 2067.

[5] K. Masuda-Jindo, Vu Van Hung and P.D. Tam, Phys. Rev. B67 (2003) 094301.

[6] K. Masuda-Jindo, S.R. Nishitami and V.V. Hung, Phys. Rev. B70 (2004) 184122.

[7] Wang F.E., Bonding Theory for metals and Alloys, (Elsevier, 2005).

[8] Wang X., Scandolo S. and Car R., Phys. Rev. Lett., (2005) 185701.

[9] Frenkel D. and Smit B., Understanding molecular Simulation: from algorithms to Applications, Computational Science Sevies Volume 1 (Academic Press, 2002).

[10] Y. Ida, Phys. Rev. B1 (1970) 2488.

[11] H. Schlosser, P. Vinet and J. Ferrante, Phys. Rev. B40 (1989) 5929.

[12] Mazomedov M.N., J. Fiz. Khimic. 61 (1987) 1003.

[13] E.I.U. Tonkov, Phase Transformation Connection in High Pressure (Metalurghia, Moskva, 1988) in Russian.

[14] A. Fernandez-Guillermet, and P. Gustafson, High temperature-high pressure, 16 (1985) 591.

Received 08 August 2010. 